alle dasselbe Definitionsgebiet $\mathbb{P}_{n}\left(M_{1}\right)$ und deren Normen lassen dort wegen (36) eine gemeinsame Schranke zu.

Mithin läßt sich eine (mitsamat den Ableitungen bis zur Ordnung $n+2$ einschlieblich) konvergente Folge $u_{h_{g}}$ herausgreifen; die Grenzfunktion löst (4) ${ }^{47}$ ).

4) Systeme von quasilinearen Differentialgieichungen konnen nach derselben Methode erledigt werden.

Es können auch in den Koeffizienten Integralo vorkommen, z. B. $\int z_{s} d x_{l}$, $\int \frac{\partial z_{s}}{\partial x_{k}} d x_{i}$ etc. Da nun eine beliebige nichtlineare Differentialgleichung durch Differentiation in ein solches immer lösbares quasilineares System ubergeht, so ware im allgemeinen Fulle nur noch zu zeigen, dab die Lösang diesen letzten Syatems der ureprïnglichen Gleichang aquivalent ist.

Lwów, 14 November 1934.

\section{Sur une propriété de la droite.}

Par

$$
\text { W. Si erpiński (Varsovie). }
$$

Une conséquence immédiate d'une proposition que j'ai démontré dans le t. XXI des Fundamenta Mathematicae, p. $39^{1}$ ) est le théorème suivant:

Si $2^{*_{0}}=\aleph_{1}$, il existe un ensemble plan. $E$, tel que le plan est une somme de $2^{t_{0}}$ ensembles disjoints, dont chacun est superposable par translation avec $E$ et en même temps le plan est une somme d'une infinité dénombrable d'ensembles, dont chacun est superposable par translation ou rotation avec $E$.

Le but de cette Note est de démontrer qu'un théorême de ce genre ne subsiste pas pour la droite. Je prouverai notamment (sans admetre que $2^{\mathrm{k}_{0}}=\mathrm{s}_{1}$ ) ce

Théorème: Soit $\mathrm{m}$ un nombre cardinal donné quelconque. Si la droite est décomposée en $\mathrm{m}$ ensembles disjoints dont chacun est superposable par translation avec un ensemble $E$, la droite n'est pas une somme de moins que $\mathrm{m}$ ensembles, dont chacun soit superposable par translation ou rotation avec $E$.

Démonstration.

Soit $m$ un nombre cardinal donné et soit $E$ un ensemble linéaire tel que l'ensemble $D$ de tous les points de la droite (axe d'abscisses) est une somme de $m$ ensembles disjoints, dont chacun est superposable par translation avec l'ensemble $E$. II existe donc un ensemble de nombres réels $M$ de puissance $m$, tel que

$$
D=\sum_{a \in M} E(a)
$$

$$
E(a) E(b)=0 \text { pour } \quad a \in M, b \in M, a \neq b,
$$

1) Cf. aussi mon livre Hypothèse du continu (Warasawa 1934, Monografje Matematycsne t. IV), p. 100. 
où $E(a)$ désigne la translation de l'ensemble $E$ de longueur $a$ (c'est-à-dire l'ensemble de tous les nombres réels $x$, tels que $x-a \in E)$ et où la sommation $\Sigma$ s'étend à tons les éléments $a$ de $M_{\text {a }}$

$$
a \in M
$$

Admettons, d'autre part, que la droite soit une somme de moins que $\mathfrak{m}$ ensembles, dont chacun est superposable par translation on rotation avec l'ensemble $E$, Désignons par $E^{*}$ l'ensemble symétrique de $E$ par rapport au point 0 . Il existe done deux ensembles de nombres réels $N_{1}$ et $N_{2}$, tels que $\overline{\overline{N_{1}}}+\overline{\overline{N_{2}}}<\mathbf{m}$ et

$$
D=\sum_{b \in N_{1}} E(b)+\sum_{b \in N_{2}} E^{*}(b) \text {. }
$$

D'après (3) on a évidemment pour a réels, et, en particulier, pour $a \in M$ :

d'où

$$
D=\sum_{b \in N_{1}} E(b+a)+\sum_{b \in N_{1}} E^{*}(b+a)
$$

$$
0 \in \sum_{b \in N_{1}} E(b+a)+\sum_{b \in N_{2}} E^{*}(b+a) \text { pour } a \in M .
$$

La somme figurant dans la formule (4) contenant $\overline{\bar{N}}_{1}+\overline{\bar{N}}_{8}<m$ termes, on conclut, d'après $\overline{\bar{M}}=\mathbf{m}$, qu'il existe deux nombres distincts de $M$, soient $a_{1}$ et $a_{2}$, tels que le nombre 0 appartient pour $a=a_{1}$ et pour $a=a_{2}$ au même terme de cette somme, c'est-à-dire quil existe un nombre $b \in N_{1}+N_{2}$, tel qu'on a ou bien

$$
b \in N_{1}, \quad 0 \in E\left(b+a_{1}\right) \text { et } 0 \in E\left(b+a_{2}\right) \text {, }
$$

ou bien

(6)

$$
b \in N_{9}, \quad 0 \in E^{*}\left(b+a_{1}\right) \text { et } 0 \in E^{*}\left(b+a_{s}\right)
$$

(les formules $(b)$ et (6) pouvant d'ailleurs être vraies à la fois). Or, d'après. (2) et $a_{1} \neq a_{2}$, on a $E\left(a_{1}\right) E\left(a_{2}\right)=0$, donc aussi $E^{*}\left(a_{1}\right) E^{*}\left(a_{2}\right)=0$, ce qui donne évidemment pour tout $b$ réel:

$$
E\left(b+a_{1}\right) E\left(b+a_{2}\right)=0 \text { et } E^{*}\left(b+a_{1}\right) E^{*}\left(b+a_{2}\right)=0 \text {, }
$$

ce qui est imcompatible avec chacune des formules (5) et (6).

L'hypothèse que la droite est une somme de moins que $m$ ensembles, dont chacun est superposable par translation ou rotation avec $E$, implique donc une contradiction.

Notre théorème est ainsi démontré.

\section{Quelques rétractes singuliers.}

Pur

\author{
Karol Borsuk (Warszawa).
}

Dans cette Note je m'occupe d'une simple opération sur les ensembles compacts en soi qui nous conduit à la construction des rétractes absolus et des rétractes absolus de voisinage ${ }^{1}$ ) dont les propriétés topologiques montrent des singularités remarquables ").

1. Soient $A$ et $B$ deux ensembles métriques, compacts en soi, disjoints et $f$ une fonction continue définie dans un sous-ensemble fermé $C$ de $A$ aux valeurs appartenants à $B$. Nous avrrivons, bien entendu, à une décomposition semi-continue ${ }^{3}$ ) de l'ensemble $A+B$ en considérant comme tranches les ensembles de la forme $\left.(x)+f^{-1}(x)^{4}\right)$, où $x \in f(C)$, et les points individuels de $A+B-(C+f(C))$. Cette décomposition sera designée dans la suite par $(A, B, f)$ et son hyper-espace par $A+B$. La fonction qui fait correspondre à tout $x \in A+B$ la tranche de la décomposition $(A, B, f)$ contenante $x$ sera désignée par $\Phi_{A, B, f}(x)$; elle transforme $A+B$ en $A+B$ d'une manière continue ${ }^{5}$ ) en transformant l'ensemble $A-C$ et aussi l'en-

1) L'ensemble $A \subset E$ est dit rétracte de $E$, lorsqu'il existe ane fonction continue $f$ définio dans $E$ ot satisfaisant aux conditions: $1^{\circ} f(E)=A, 2^{\circ} f(x)=x$ pour tout $x \in A$. L'ensemble métrique et compact en soi $A$ est dit rétracte absolu (rosp. rétracte absolu de voisinage) si, pour tont espace métrique $M \supset A$, l'oneemble $E$ est an rétracte de $M$ (resp. un rétracte d'un certajn roisinage de $A$ dans $\boldsymbol{M}$ ).

a) Les exemples considerés dans cette Note sont construits pour répondro à certains problèmies posés par M. C. Ku ra to ws ki concernant la caractérisation des espaces péaniens en dimension $n^{\natural}$ par des propriótés intrinsèques. Voir la Note de M. C. K $\mathrm{aratow} 8 \mathrm{ki}$ : Sur les espaces localement connexes et péaniens en dimensions $n$, ce volume, p. 267-287.

3) Quant la définition d'une d́composition semi-continue ot son hyperespace roir C. Ku ratow aki, Fund. Math. 11 (1928), p. 169-185.

4) $f^{-1}(y)$ désigne l'ensemble de, tous les $x$ tels que $f(x)=y$.

s) C. Karatowki, 1. c. p. 173, th. III. 